\title{
Information Security through Data Encryption and Data Hiding
}

\author{
Samir Kumar Bandyopadhyay ${ }^{1}$ and Somaditya Roy ${ }^{2}$ \\ ${ }^{1}$ Department of Computer Science \& Engineering \\ ${ }^{2}$ A.K.Choudhuri School of Information Technology \\ University of Calcutta, India
}

\begin{abstract}
This paper introduces a model for information security and message hiding based on image steganography as well as it describes about various steps of that proposed model in brief.
\end{abstract}

\section{Keywords-Image steganography, Data Hiding}

\section{INTRODUCTION}

Information Technology is the most essential aspect in today's world. Based on this fact computer application is still developing to handle securely the financial as well as the personal data more effectively. These data are extremely important from every aspect and we need to secure this from unauthorized access. Security is the process of preventing and detecting unauthorized use of data or computer or network. Prevention measures help us to stop unauthorized users from accessing any part of computer system. Detection helps to determine whether or not someone attempted to break into the system, if they were successful, and what they may have done. To achieve that security we may use various cryptography techniques. However, today data encryption is not everything or we cannot achieve strong security through this, we also need to secure the presence of date. Here comes the necessity of steganography. But we need to remember that neither cryptography nor steganography gives the total security to a data or information uniquely, thus we need to apply both techniques to achieve essential security. There are number of ways this can be done, but here we will concern with methods of altering the information in such a way that the recipient can undo the alteration and discover the original text.

In this paper, we concentrate on developing an innovative image steganographic model for information security through data encryption as well as data hiding.

\section{MODEL}

This is an image steganographic technique for message hiding as well as message encryption and decryption. In our model we use finger impression or finger image for stego

image, but we can also implement it by iris impression or face impression or same.
The basic idea of our model is a sender encrypts a message by his/her fingerprint to create a stegoimage and send it to the intended receiver. Receiver uses sender's public key to decrypt it to get the original message. Here, the finger impressions of users are act like private keys. So, in this model we need to pair of keys or two pair of images as public and private key. Initially we take $8 \mathrm{X} 8$ pixel JEPG (gray scale) finger impressions for private key. Now, we need to calculate the ASCII values of each pixel of finger impressions and put it in an $8 \times 8$ matrix.

Then, we consider the prime numbers between 0-255 which are $2,3,5,7,11,13,17,19,23,29,31,37,41,43,47,53,59$, $61,67,71,73,79,83,89,97,101,103,107,109,113,127$, $131,137,139,149,151,157,163,167,173,179,181,191$, 193, 197, 199, 211, 223, 227, 229, 233, 239, 241 and 251. After that we calculate the difference between the ASCII value and the nearest prime number of it's and form another 8X8 matrix and put difference values into it in binary form. I.e. if we consider the ASCII 100 than its nearest prime number which is 101 then the difference will be +1 , again if we consider the ASCII 243 than its nearest prime number which is 241 then the difference will be -2 and so on. There may the case where the number will lie exactly in the middle of two prime numbers. Let us consider the ASCII 60. Now, in this case the nearest prime numbers are 59 as well as 61 both. In such case we take it as +1 . After constructing the matrix we convert it to its corresponding 8 X8 JEPG gray scale image and it will be our public key or share key.

Now, for encrypt a message we can follow either of two ways. Either we can encrypt the original message by using our private key and send it to the intended receiver or we can encrypt the message by using receiver public key and send it to him/her. Let us take the second possibility. In this case we need to insert the message in LSB of each pixels of our public image. After inserting the whole message we have to look farther to determine the changing pixels positions. For data hiding and data sending we need a cover image. We have no restriction of choosing cover image; we just need to reflect that changing information into it. But to insert that particular information we have to consider the following cases: 
Case 1: (Number of change in pixel >

$$
\text { Number of unchanged pixel) }
$$

Then, image.

Change the Cover Image in the row wise as the public

If (Number of change in pixel > Number of unchanged pixel)

Change the LSB of unchanged position pixel)

If (Number of change in pixel $<=$ Number of unchanged

Change the 2nd last LSB of changed position

Case 2: (Number of change in pixel <=

Number of unchanged pixel)

Then, image.

Change Cover Image in Column wise as the public pixel)

If (Number of change in pixel > Number of unchanged

Change the LSB of unchanged position

If (Number of change in pixel <= Number of unchanged pixel)

Change the 2nd last LSB of changed position

As the whole information is been reflected in the cover image we are ready to send it to the intended receiver.

In the receiver end we need the private key or the private image of the corresponding sender to get the actual message. One can only get the information of the change or the unchanged positions in pixels through steganalysis on cover image but not ever able to decrypt it into the actual message without having the private image or private key. After steganalysis we get the information of the position of changed or unchanged pixels. In our case, we encrypt the message using public image so we need to decrypt it using private image. Now, the interesting facts of our particular model is that we insert the message in LSB of our private image and then we look for the change and unchanged positions of pixels, if we perform the some in public image we can observe that it is perfectly opposite of private image. I.e. all the change positions in private image are totally unchanged in public image and all the unchanged positions of private image are totally changed in public image. So, at the receiver end after getting the changed and unchanged position's information we needed to update LSB of the public image in opposite fashion. The changed position remains unchanged and the unchanged position will need to change. After this updating is done we simply consider the LSB in serial manner to get the original message. Now, public image can be same for different users as we take only the ASCII and prime difference but none can decrypt it properly without having the actual private image. But Private images cannot be same in any case as we take the finger impression.

\section{ANALYSIS}

Let our Original Message: Good Boy

ASCII Value : 71, 111, 111, 100, 32, 66, 111, 121

Binary Representation: 01000111, 01101111, 01101111, 01100100, 00100000, 01000010, 01101111, 01111001.

We take public image for message encryption.

Public Image + Original Message (Update LSB of public image either by 1 or by 0 depending on message)

$=$ Initial Stego Image

\section{Private Image}

8X8 JEPG grey scale Finger impression or private image.

A = Private key or private image

\begin{tabular}{|c|c|c|c|c|c|c|c|}
\hline 255 & 255 & 244 & 168 & 173 & 245 & 255 & 254 \\
\hline 255 & 251 & 145 & 101 & 84 & 142 & 255 & 255 \\
\hline 254 & 216 & 93 & 110 & 95 & 98 & 240 & 255 \\
\hline 255 & 197 & 93 & 97 & 95 & 84 & 227 & 254 \\
\hline 255 & 187 & 86 & 81 & 75 & 76 & 225 & 254 \\
\hline 254 & 201 & 61 & 68 & 63 & 86 & 240 & 255 \\
\hline 255 & 245 & 100 & 60 & 64 & 131 & 255 & 253 \\
\hline 255 & 255 & 232 & 170 & 178 & 243 & 255 & 255 \\
\hline
\end{tabular}

Figure 1. ASCII value of each pixels of private image.

B = Public key or public image

\begin{tabular}{|l|l|l|l|l|l|l|l|}
\hline-4 & -4 & -3 & -1 & 0 & -4 & -4 & -3 \\
\hline-4 & 0 & +4 & 0 & -1 & -3 & -4 & -4 \\
\hline-3 & -5 & +4 & -1 & +2 & -1 & +1 & -4 \\
\hline-4 & 0 & +4 & 0 & +2 & -1 & 0 & -3 \\
\hline-4 & +4 & +3 & +2 & -2 & +3 & 2 & -3 \\
\hline-3 & -2 & 0 & -1 & -2 & +3 & 1 & -4 \\
\hline-4 & -4 & +1 & +1 & +3 & 0 & -4 & -2 \\
\hline-4 & -4 & +1 & +3 & +1 & -2 & -4 & -4 \\
\hline
\end{tabular}

ASCII to nearest prime.
Figure 2. V alues of difference between 


\begin{tabular}{|c|c|c|c|c|c|c|c|}
\hline 5 & 5 & 12 & 34 & 74 & 96 & 105 & 120 \\
\hline 27 & 38 & 64 & 96 & 140 & 183 & 202 & 192 \\
\hline 53 & 28 & 16 & 7 & 42 & 32 & 56 & 127 \\
\hline 60 & 56 & 33 & 0 & 55 & 41 & 98 & 80 \\
\hline 120 & 124 & 70 & 35 & 25 & 55 & 191 & 183 \\
\hline 150 & 159 & 128 & 102 & 106 & 152 & 177 & 161 \\
\hline 158 & 145 & 144 & 153 & 162 & 152 & 155 & 185 \\
\hline 178 & 162 & 163 & 165 & 178 & 193 & 213 & 221 \\
\hline
\end{tabular}

\begin{tabular}{|l|l|l|l|l|l|l|l|}
\hline 1100 & 1101 & 1100 & 1110 & 0000 & 1100 & 1101 & 1101 \\
\hline 1100 & 0001 & 0101 & 0000 & 1111 & 1101 & 1101 & 1101 \\
\hline 1100 & 1011 & 0101 & 1110 & 0011 & 1111 & 0001 & 1101 \\
\hline 1100 & 0001 & 0101 & 0000 & 0010 & 1111 & 0000 & 1100 \\
\hline 1100 & 0100 & 0011 & 0010 & 1110 & 0010 & 0010 & 1100 \\
\hline 1100 & 1111 & 0000 & 1110 & 1110 & 0010 & 0001 & 1100 \\
\hline 1100 & 1101 & 0001 & 0000 & 0011 & 0001 & 1101 & 1111 \\
\hline 1100 & 1101 & 0001 & 0011 & 0001 & 1110 & 1100 & 1101 \\
\hline
\end{tabular}

Figure 3. Binary representation of above.

In our case, we take public image for message encryption. Public image + Original Message (Update LSB of public image either by 1 or by 0 depending on message) $=$ Initial Stego Image

Figure 4. After inserting the whole message to the above table.

Determine the positions of changed pixel in public image:

\begin{tabular}{rrrr}
{$[0,1][0,2][0,3]$} & {$[0,5][0,6]$} & $=$ change in 5 pixels \\
{$[1,1][1,2]$} & {$[1,6][1,7]$} & $=$ change in 4 pixels \\
{$[2,2][2,3][2,4]$} & {$[2,7]$} & $=$ change in 5 pixels \\
{$[2,0]$} & {$[3,7]$} & $=$ change in 3 pixels \\
{$[3,1][3,2]$} & {$[4,5]$} & {$[4,7]$} & $=$ change in 2 pixels \\
& & $=$ change in 4 pixels \\
{$[5,0][5,1]$} & {$[5,3]$} & {$[5,5]$} & \\
{$[6,1]$} & {$[6,3]$} & {$[6,5][6,6][6,7]$} & $=$ change in 5 pixels \\
{$[7,1]$} & & {$[7,7]$} & $=$ change in 2 pixels \\
\hline
\end{tabular}

Total Change $=30$ pixels

Here, number of changed in pixel positions $=30$

Therefore, number of unchanged positions $=(64-30)=34$ That means, Number of change in pixel positions> Number of unchanged pixel positions So, we change the cover image in column wise.

\begin{tabular}{|l|l|l|l|l|l|l|l|}
\hline 1100 & 1100 & 1101 & 1111 & 0000 & 1100 & 1100 & 1101 \\
\hline 1100 & 0000 & 0100 & 0000 & 1111 & 1101 & 1100 & 1100 \\
\hline 1101 & 1011 & 0100 & 1111 & 0010 & 1111 & 0001 & 1100 \\
\hline 1100 & 0000 & 0100 & 0000 & 0010 & 1111 & 0000 & 1101 \\
\hline 1100 & 0100 & 0011 & 0010 & 1110 & 0011 & 0010 & 1101 \\
\hline 1101 & 1110 & 0000 & 1111 & 1110 & 0011 & 0001 & 1100 \\
\hline 1100 & 1100 & 0001 & 0001 & 0011 & 0000 & 1100 & 1110 \\
\hline 1100 & 1100 & 0001 & 0011 & 0001 & 1110 & 1100 & 1100 \\
\hline
\end{tabular}

8X8 JEPG grey scale Cover Image.

$\mathrm{C}=$

Figure 5. ASCII value of each pixels of cover image.

\begin{tabular}{|l|l|l|l|l|l|l|l|}
\hline 0000 & 0000 & 0000 & 0010 & 0100 & 0110 & 0110 & 0111 \\
0101 & 0101 & 1100 & 0010 & 1010 & 0000 & 1001 & 1000 \\
\hline 0001 & 0010 & 0100 & 0110 & 1000 & 1011 & 1100 & 1100 \\
1011 & 0110 & 0000 & 0000 & 1100 & 0111 & 1010 & 0000 \\
\hline 0011 & 0001 & 0001 & 0000 & 0010 & 0010 & 0011 & 0111 \\
0101 & 1100 & 0000 & 0111 & 1010 & 0000 & 1000 & 1111 \\
\hline 0011 & 0011 & 0010 & 0000 & 0011 & 0010 & 0110 & 0101 \\
1100 & 1000 & 0001 & 0000 & 0111 & 1001 & 0010 & 0000 \\
\hline 0111 & 0111 & 0100 & 0010 & 0001 & 0011 & 1011 & 1011 \\
1000 & 1100 & 0110 & 0011 & 1001 & 0111 & 1111 & 0111 \\
\hline 1001 & 1001 & 1000 & 0110 & 0110 & 1001 & 1011 & 1010 \\
0110 & 1111 & 0000 & 0110 & 1010 & 1000 & 0001 & 0001 \\
\hline 1001 & 1001 & 1001 & 1001 & 1010 & 1001 & 1001 & 1011 \\
1110 & 0001 & 0000 & 1001 & 0010 & 1000 & 1011 & 1001 \\
\hline 1011 & 1010 & 1010 & 1010 & 1011 & 1100 & 1101 & 1101 \\
0010 & 0010 & 0011 & 0101 & 0010 & 0001 & 0101 & 1101 \\
\hline
\end{tabular}

Figure 6. Binary representation of the above.

\section{Change row wise:}

\begin{tabular}{|l|l|l|l|l|l|l|l|}
\hline 0000 & 0000 & 0000 & 0010 & 0100 & 0110 & 0110 & 0111 \\
0100 & 0101 & 1100 & 0010 & 1011 & 0000 & 1001 & 1001 \\
\hline 0001 & 0010 & 0100 & 0110 & 1000 & 1011 & 1100 & 1100 \\
1011 & 0100 & 0010 & 0000 & 1100 & 0111 & 1000 & 0010 \\
\hline 0011 & 0001 & 0001 & 0000 & 0010 & 0010 & 0011 & 0111 \\
0101 & 1101 & 0000 & 0111 & 1010 & 0001 & 1001 & 1111 \\
\hline 0011 & 0011 & 0010 & 0000 & 0011 & 0010 & 0110 & 0101 \\
1100 & 1010 & 0011 & 0000 & 0111 & 1001 & 0010 & 0010 \\
\hline 0111 & 0111 & 0100 & 0010 & 0001 & 0011 & 1011 & 1011 \\
1000 & 1100 & 0110 & 0011 & 1001 & 0101 & 1111 & 0101 \\
\hline 1001 & 1001 & 1000 & 0110 & 0110 & 1001 & 1011 & 1010 \\
0100 & 1101 & 0000 & 0100 & 1010 & 1010 & 0001 & 0001 \\
\hline 1001 & 1001 & 1001 & 1001 & 1010 & 1001 & 1001 & 1011 \\
1111 & 0001 & 0001 & 1001 & 0000 & 1000 & 1011 & 1001 \\
\hline 1011 & 1010 & 1010 & 1010 & 1011 & 1100 & 1101 & 1101 \\
0010 & 0000 & 0011 & 0101 & 0010 & 0001 & 0101 & 1111 \\
\hline
\end{tabular}

Figure 7. Binary representation of the above after inserting the information of change in pixels positions in rowwise.

\section{Cover Image}


Change column wise:

\begin{tabular}{|l|l|l|l|l|l|l|l|}
\hline 0000 & 0000 & 0000 & 0010 & 0100 & 0110 & 0110 & 0111 \\
0101 & 0101 & 1110 & 0000 & 1010 & 0010 & 1011 & 1001 \\
\hline 0001 & 0010 & 0100 & 0110 & 1000 & 1011 & 1100 & 1100 \\
1011 & 0110 & 0010 & 0000 & 1100 & 0111 & 1000 & 0000 \\
\hline 0011 & 0001 & 0001 & 0000 & 0010 & 0010 & 0011 & 0111 \\
0111 & 1101 & 0010 & 0101 & 1000 & 0000 & 1000 & 1111 \\
\hline 0011 & 0011 & 0010 & 0000 & 0011 & 0010 & 0110 & 0101 \\
1100 & 1000 & 0011 & 0000 & 0111 & 1001 & 0010 & 0000 \\
\hline 0111 & 0111 & 0100 & 0010 & 0001 & 0011 & 1011 & 1011 \\
1000 & 1101 & 0110 & 0011 & 1001 & 0101 & 1111 & 0111 \\
\hline 1001 & 1001 & 1000 & 0110 & 0110 & 1001 & 1011 & 1010 \\
0100 & 1111 & 0000 & 0100 & 1010 & 1010 & 0001 & 0000 \\
\hline 1001 & 1001 & 1001 & 1001 & 1010 & 1001 & 1001 & 1011 \\
1110 & 0001 & 0000 & 1011 & 0010 & 1010 & 1001 & 1001 \\
\hline 1011 & 1010 & 1010 & 1010 & 1011 & 1100 & 1101 & 1101 \\
0010 & 0010 & 0011 & 0101 & 0010 & 0001 & 0101 & 1101 \\
\hline
\end{tabular}

Figure 8. Binary representation of the above after inserting the information of change in pixels positions in columnwise.

Unchanged pixel position: Green

Changed pixel positions: Blue

Here, we change pixels of the cover image in column wise.

$\mathrm{C} 1=$

\begin{tabular}{|c|c|c|c|c|c|c|c|}
\hline 5 & 5 & 14 & 36 & 74 & 98 & 107 & 121 \\
\hline 27 & 38 & 66 & 96 & 140 & 183 & 204 & 192 \\
\hline 55 & 29 & 18 & 9 & 44 & 32 & 56 & 127 \\
\hline 60 & 56 & 35 & 0 & 55 & 41 & 98 & 80 \\
\hline 120 & 125 & 70 & 35 & 25 & 57 & 191 & 183 \\
\hline 148 & 159 & 128 & 104 & 106 & 154 & 177 & 162 \\
\hline 158 & 145 & 144 & 155 & 162 & 154 & 157 & 185 \\
\hline 178 & 162 & 163 & 165 & 178 & 193 & 213 & 221 \\
\hline
\end{tabular}

Figure 9. After inserting the information of change in pixels positions in columnwise.

$\mathrm{C} 1$ is the expected final stego image which is ready to send to the intended receiver.

Now, if we insert the message in LSB of private image we will get the following:

\begin{tabular}{|l|l|l|l|l|l|l|l|}
\hline $\mathbf{1 1 1 1}$ & 1111 & 1111 & 1010 & $\mathbf{1 0 1 0}$ & 1111 & 1111 & $\mathbf{1 1 1 1}$ \\
$\mathbf{1 1 1 0}$ & 1111 & 0100 & 1000 & $\mathbf{1 1 0 0}$ & 0101 & 1111 & $\mathbf{1 1 1 1}$ \\
\hline $\mathbf{1 1 1 1}$ & 1111 & 1001 & $\mathbf{0 1 1 0}$ & $\mathbf{0 1 0 1}$ & $\mathbf{1 0 0 0}$ & 1111 & 1111 \\
$\mathbf{1 1 1 0}$ & 1011 & 0001 & $\mathbf{0 1 0 0}$ & $\mathbf{0 1 0 1}$ & $\mathbf{1 1 1 1}$ & 1111 & 1111 \\
\hline 1111 & $\mathbf{1 1 0 1}$ & 0101 & 0110 & 0101 & $\mathbf{0 1 1 0}$ & $\mathbf{1 1 1 1}$ & 1111 \\
1110 & $\mathbf{1 0 0 1}$ & 1101 & 1110 & 1111 & $\mathbf{0 0 1 1}$ & $\mathbf{0 0 0 1}$ & 1111 \\
\hline $\mathbf{1 1 1 1}$ & 1100 & 0101 & $\mathbf{0 1 1 0}$ & $\mathbf{0 1 0 1}$ & $\mathbf{0 1 0 1}$ & $\mathbf{1 1 1 0}$ & 1111 \\
$\mathbf{1 1 1 0}$ & 0101 & 1101 & $\mathbf{0 0 0 0}$ & $\mathbf{1 1 1 0}$ & $\mathbf{0 1 0 1}$ & $\mathbf{0 0 1 0}$ & 1110 \\
\hline $\mathbf{1 1 1 1}$ & $\mathbf{1 0 1 1}$ & $\mathbf{0 1 0 1}$ & $\mathbf{0 1 0 1}$ & $\mathbf{0 1 0 0}$ & 0100 & $\mathbf{1 1 1 0}$ & 1111 \\
$\mathbf{1 1 1 0}$ & $\mathbf{1 0 1 0}$ & $\mathbf{0 1 1 1}$ & $\mathbf{0 0 0 0}$ & $\mathbf{1 0 1 0}$ & 1100 & $\mathbf{0 0 0 0}$ & 1110 \\
\hline 1111 & 1100 & $\mathbf{0 0 1 1}$ & 0100 & $\mathbf{0 0 1 1}$ & 0101 & $\mathbf{1 1 1 1}$ & $\mathbf{1 1 1 1}$ \\
1110 & 1001 & $\mathbf{1 1 0 0}$ & 0100 & $\mathbf{1 1 1 0}$ & 0110 & $\mathbf{0 0 0 1}$ & $\mathbf{1 1 1 0}$ \\
\hline $\mathbf{1 1 1 1}$ & 1111 & $\mathbf{0 1 1 0}$ & 0011 & $\mathbf{0 1 0 0}$ & 1000 & 1111 & 1111 \\
$\mathbf{1 1 1 0}$ & 0101 & $\mathbf{0 1 0 1}$ & 1100 & $\mathbf{0 0 0 1}$ & 0011 & 1111 & 1101 \\
\hline $\mathbf{1 1 1 1}$ & 1111 & $\mathbf{1 1 1 0}$ & $\mathbf{1 0 1 0}$ & $\mathbf{1 0 1 1}$ & $\mathbf{1 1 1 1}$ & $\mathbf{1 1 1 1}$ & 1111 \\
$\mathbf{1 1 1 0}$ & 1111 & $\mathbf{1 0 0 1}$ & $\mathbf{1 0 1 1}$ & $\mathbf{0 0 1 1}$ & $\mathbf{0 0 1 0}$ & $\mathbf{1 1 1 0}$ & 1111 \\
\hline
\end{tabular}

Figure 10. After inserting the message in LSB of the private image.

Determine the positions of changed pixel in private image:

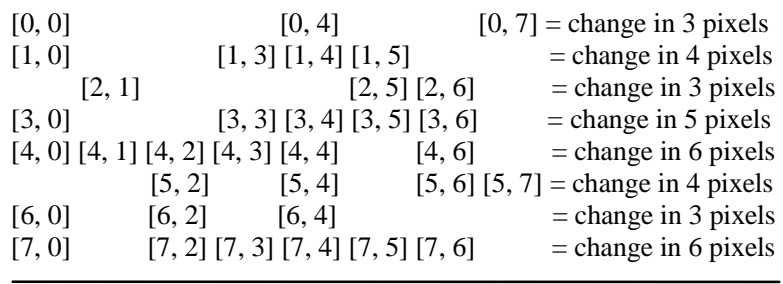

Total Change $=34$ pixels

We can observe that the positions of changed pixels in public image are perfectly the opposite in case of private image. So, in the receiver end after we get the stego image first we need to perform steganalysis on it to get the information of positions of changed and unchanged pixels. As soon we get that we just required inserting that in LSB of private image but in opposite manner. After inserting the whole information if we consider the LSBs together of the private image we will get the original message.

\section{CONCLUSIION}

In our model we sincerely consider all the basic principles of information security and try to keep it. As we use two different images one for private and another for public key it gives us to preserve the authentication and helps to overcome non-repudiation issue. In the actual carrier which is cover image in our case, we only send the minimal information of the changes in pixel positions of the key instead of the actual message and none can decrypt or retrieve the original message from it without knowing any knowledge of key. It gives us the privileged to sincerely keep the confidentiality of data or message. This model is very simple to implement yet very complicated to break. Initially we construct this model on image steganography with simple gray scale impression but there is a larger scope to implement it in other steganographic techniques also in future.

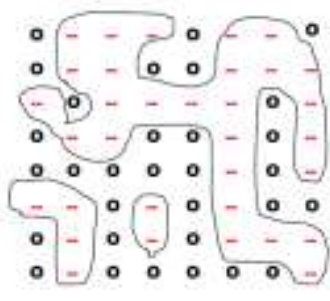

(A)

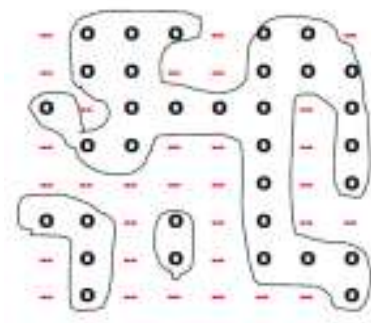

(B)
Figure 11. (A) Private Image, (B) Public Image 
Unchanged pixel position:

Changed pixel positions:

\section{ACKNOWLEDGMENT (HEADING 5)}

The preferred spelling of the word "acknowledgment" in America is without an "e" after the "g". Avoid the stilted expression, "One of us (R. B. G.) thanks . . ." Instead, try "R. B. G. thanks". Put sponsor acknowledgments in the unnumbered footnote on the first page.

\section{REFERENCES}

[1] G. Eason, B. Noble, and I. N. Sneddon, "On certain integrals of Lipschitz-Hankel type involving products of Bessel functions," Phil. Trans. Roy. Soc. London, vol. A247, pp. 529-551, April 1955. (references)

[2] J. Clerk Maxwell, A Treatise on Electricity and Magnetism, 3rd ed., vol. 2. Oxford: Clarendon, 1892, pp.68-73.

[3] I. S. Jacobs and C. P. Bean, "Fine particles, thin films and exchange anisotropy," in Magnetism, vol. III, G. T. Rado and H. Suhl, Eds. New York: Academic, 1963, pp. 271-350.

[4] K. Elissa, "Title of paper if known," unpublished.
[5] R. Nicole, "Title of paper with only first word capitalized," J. Name Stand. Abbrev., in press.

[6] Y. Yorozu, M. Hirano, K. Oka, and Y. Tagawa, "Electron spectroscopy studies on magneto-optical media and plastic substrate interface," IEEE Transl. J. Magn. Japan, vol. 2, pp. 740-741, August 1987 [Digests 9th Annual Conf. Magnetics Japan, p. 301, 1982].

[7] M. Young, The Technical Writer's Handbook. Mill Valley, CA: University Science, 1989.

\section{AUTHORS PROFILE}

Authors Profile ...

\section{Authors:}

Prof. Samir Kumar Bandyopadhyay did his B.E., M.Tech., Ph. D (ComputerScience \& Engineering), His research interests include Bio-medical Engg, Mobile Computing, Pattern Recognition, Graph Theory, Software Engg.,etc. He is Senior Member of IEEE.

Mr. Somaditya Roy is presently working as Research Scholar in the Department of Computer Science and Engineering, University of Calcutta, India. 